\title{
Exploring fine-grained sentiment values in online product reviews
}

\author{
Phoey Lee Teh \\ Department of Computing and Information Systems \\ Sunway University \\ Bandar Sunway, Malaysia \\ phoeyleet@sunway.edu.my \\ Irina Pak \\ Department of Computing and Information Systems \\ Sunway University \\ ipak1992@gmail.com
}

\begin{abstract}
We hypothesise that it is possible to determine a fine-grained set of sentiment values over and above the simple three-way positive/neutral/negative or binary Like/Dislike distinctions by examining textual formatting features. We show that this is possible for online comments about ten different categories of products. In the context of online shopping and reviews, one of the ways to analyse consumers' feedback is by analysing comments. The rating of the "like" button on a product or a comment is not sufficient to understand the level of expression. The expression of opinion is not only identified by the meaning of the words used in the comments, nor by simply counting the number of "thumbs up", but it also includes the usage of capital letters, the use of repeated words, and the usage of emoticons. In this paper, we investigate whether it is possible to expand up to seven levels of sentiment by extracting such features. Five hundred questionnaires were collected and analysed to verify the level of "like" and "dislike" value. Our results show significant values on each of the hypotheses. For consumers, reading reviews helps them make better purchase decisions but we show there is also value to be gained in a finer-grained sentiment analysis for future commercial website platforms.
\end{abstract}

\section{Keywords - value expression; like and dislike; sentiment value}

\section{INTRODUCTION}

The impact of E-commerce over the internet has significantly supported the global economy. For instance: in the past eight years, Khoury and Shirmohammadi [1] analyzed the online shopping experience. They developed a system that allowed customers to share their online shopping experience, and thus also enquire about product's opinion. Lin and Yan [2] stated that many brick-and-mortar stores have extended their business online (e.g. to Tao Bao), due to its online convenience and cheaper prices. Meanwhile, Shen and

\author{
Paul Rayson \\ School of Computing and Communications, \\ Lancaster University, UK. \\ p.rayson@lancaster.ac.uk \\ Scott Piao \\ School of Computing and Communications, \\ Lancaster University, UK. \\ s.piao@lancaster.ac.uk
}

Sunndaresan [3] had analyzed the network of ecommerce, its online business structure, its interactions, its reputation on eBay. Thus research has shown that ecommerce has been growing and become part of the global economy. Hossain [4] also studied the impact of ecommerce and internet on the global economy. Online shopping has become popular and widespread. In order to maintain a good online shopping experience, consumer feedback, reviews and comments are vitally important to consider.

Consumer behavior has become one of the important topics in marketing research recently. Much research has been undertaken on the topic of consumer behavior. For instance, He et al. [5] collected reviews on consumer's opinion and analyzed it. Zin et al. [6] built a behavior analyzing tool to study consumer behavior. Lin and Yan [2] stated that consumer buying behavior is important to analyse because it can help to explain 1) emotional motives of the consumer, 2) rational motivation as understanding of cost-effective and affordable price, 3 ) purchase motivation when consumer has a special preference. Fan and Sun [7] highlighted that it is important to analyze online comments. They proposed a tool for collecting comments and analyzing sentiment from collected comments.

\section{RELATED WORK}

Kim et al. [8] hypothesised that users tend to use words in capital letters in order to emphasize a particular point or word. Also they pinpointed that capital letters, emoticons, abbreviations and exclamations are used in online chatting to express non-verbal signals. Vandergriff [9] has researched on how emotions have been used in computer communication. According to his paper, capitalization might be used when user want to show "increasing volume". 
Neviarouskaya, Prendinger, and Ishizuka [10] studied interpretation (meaning) behind text messaging in online communication. They created a model which can determine emotion state. There is consideration of capital letters in their model which is in the first level of "Symbolic cue analysis module" whereby the model includes repeated punctuation, capital letters and emoticons. They stated that capital letters in sentence might be used in emphasizing words.

With the rapid growth in online social media, Pang, Lee, and Vaithyanathan [11] described the usefulness of review classification. They use Naïve Bayes, Maximum Entropy and Support Vector Machines methods to compare movie reviews and to apply sentiment classification. Thelwall et. al. [12] have also analysed social media comments. In their paper, they discussed the importance of emotional expression in informal text such as blogs, comments, forums and experimented with MySpace comments and predicted positive and negative emotions using their SentiStrength tool. Their paper describes the role of emotions in free text language, and explains how sentiment analysis can be commercially beneficial.

Prabowo and Thelwall [13] proposed the approach to examine the text though sentiment analysis on consumer reviews, feedback and comments. Their paper shows that categorizing text into positive or negative categories can bring benefits for many purposes. They presented the importance of classifying texts into a three-way sentiment model with positive, negative and neutral categories. Thelwall, Buckley, and Paltoglou [14] analysed free language text by extracting sentiment value using SentiStrength2. SentiStrength2 is a tool that is able to identify the positive or negative value of the texts.

There are many other studies that address the importance of sentiment analysis. Such as Cobb, Mays, and Craham [15] who conducted experiments to understand how the consumer's decision-making depends on online reviews and messages posted. And later, Xue, Fu, and Shaobin [16] pinpointed that there is an increasing number of users who share their feelings and emotions online, which led them to propose a model to build dictionary that able to classify text into positive, negative or neutral categories.

Liang and Dai [17] discussed the popularity of microblogging sites such as Twitter and Facebook and the significance of analyzing microblogs using sentiment. They also developed a system that was able to extract messages from social media, identifying the sentiment value into two values: positive or negative.

In other research, the formatting of different varieties of text has been studied. For example, the repetition of punctuation in the texts by Kalman and Gergle [18]. They described the repetition of punctuation by analyzing Twitter micro blogs. They concluded that users tend to use repetition of punctuation in text to express personalization and file tuning of messages like micro blogs and comments. Besides that, Urabe, Rzepka, and Araki [19] discussed how effectively emoticons can be used to express the non-verbal emotional tones and body movement They proposed an emoticon recommendation system that helps users to express emotions using emoticons.

In addition, Kalman and Gergle [20] also studied the repetition of letters in online communication, which was able to express the non-verbal cues in the texts.

Overall, understanding the detailed value of expressions has become significant with a vast body of related work in sentiment analysis but previous work has only explored twoway or three-way classifications. In our work, we hypothesized a seven-way variation of values expressed in free text language.

\section{MEthodology}

First, we collected 1,041 online comments, from ten different types of products from Social Media Platform, included Amazon, Facebook, E-bay and GSM Arena. The comments related to a group of products from various categories: 1) Beauty and Health 2) Camera 3) Computer 4) Consumer Electronics 5) Fashion 6) Home appliance 7) Jewellery and Watch 8) Mobiles and Tables 9) Sport goods and 10) Toys and Kids. The comments were analyzed with the Wmatrix system [21] to identify the word frequency profile. Seven types of expressions were categorized based on the observation of these comments. Hypotheses were formed by identifying the highest frequency words and grouping the variety of ways they were expressed.

Figure 1 shows the seven varieties of format used in expressing comments.

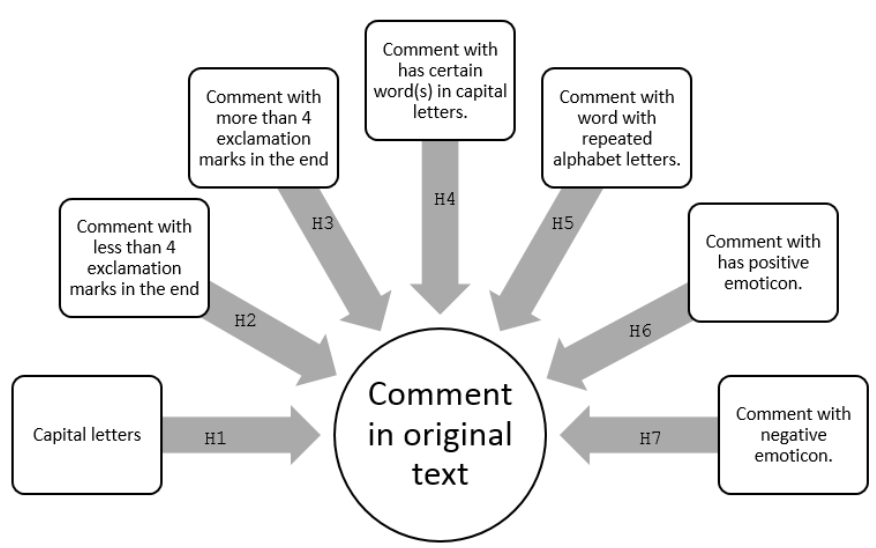

Figure 1 Comment types

In order to investigate the effect of these comment types, thirty questions on a seven-point Likert Scale ranging from 'strongly dislike' to 'strongly like' was used to rate the level of expression. Five hundred questionnaires were collected, each question with 7 varieties of formats and 1 plain unformatted 
text, the 7 varieties of comment types are used in representing the hypothesis. SPSS was used to carry out an analysis. In order to check the data accuracy, we ran Reliability Statistic test using SPSS. The accuracy of the data is $95.4 \%$.

$>$ H1: Expression of text is affected if whole comment is written in capital letters.

$>$ H2: Expression of text is affected if comment has less than 4 exclamation marks in the end.

$>$ H3: Expression of text is affected if comment has more than 4 exclamation marks in the end.

$>$ H4: Expression of text is affected if comment has certain word(s) in capital letters.

$>$ H5: Expression of text is affected if comment has word with repeated alphabet letters.

$>$ H6: Expression of text is affected if comment has positive emoticon.

$>$ H7: Expression of text is affected if comment has negative emoticon.

\section{RESULTS}

Table 1 and Table 2 show the significance of the relationships between the original text and each of the different types of text (capital letters, with emoticons etc.).

Table 1 Correlation of Positive comments

\begin{tabular}{|c|c|c|c|c|c|c|c|}
\hline Comment & $\mathrm{Hl}$ & $\mathrm{H} 2$ & H3 & H4 & H5 & H6 & H7 \\
\hline I love it & $.254^{+\infty}$ & $.295^{*+}$ & $.270^{+\infty}$ & -.003 & $.083^{*}$ & $.138^{\circ \mathrm{H}}$ & $.130^{\circ-}$ \\
\hline I like it & $.284^{+4}$ & $.289^{m+}$ & $237^{*+}$ & $-110^{*}$ & -.067 & .028 & $.130^{\circ+}$ \\
\hline I am very happy & $.290^{+\infty}$ & $.257^{\text {*n }}$ & $299^{* *}$ & $.312^{\text {+n+ }}$ & .081 & $.17 \mathrm{e}^{+\infty}$ & $324^{--}$ \\
\hline I am glad & $.32 \mathrm{~g} \mathrm{~m}^{\mathrm{m}}$ & $.310^{\mathrm{m}}$ & $.140^{n+}$ & $.191^{* *}$ & $.088^{*}$ & $.195^{*+}$ & .058 \\
\hline I am big fan & $387^{\text {*n+ }}$ & $.152^{+*}$ & $.275^{\text {n+ }}$ & $.283^{\text {*n }}$ & $.304^{*-}$ & $.227^{+\infty}$ & $.181^{*-}$ \\
\hline My favourite & $487^{*+1}$ & $.370^{+4}$ & $283^{*}$ & $292^{*+*}$ & $285^{*}$ & $301^{* *}$ & $.182^{*-}$ \\
\hline Hours of fun & $.481^{* *}$ & $40 g^{m+}$ & $250^{\circ *}$ & $.192^{*+}$ & $.146^{* *}$ & .055 & $.202^{+1+}$ \\
\hline Very satisfied & $.422^{+*}$ & $.25 \mathrm{~g}^{\mathrm{m}}$ & $.097^{\circ}$ & -.021 & -.033 & .004 & -.032 \\
\hline I prefer it & $.277^{\text {*n+ }}$ & $163^{+*}$ & .000 & .004 & $-117^{*+\infty}$ & .046 & $-.402^{+\infty}$ \\
\hline Really enjoy & $.436^{+\infty}$ & $.284^{+n}$ & $240^{+*}$ & .083 & $.088^{*}$ & $.146^{+\infty}$ & $.122^{+*}$ \\
\hline I recommend it & $.385^{+*}$ & $.357^{\text {*n }}$ & $218^{*+}$ & $.158^{+n}$ & $.088^{*}$ & .015 & -.056 \\
\hline Exceed expectations & $.383^{+*}$ & $204^{+*+}$ & $297^{*+}$ & .014 & .045 & .043 & $.108^{*}$ \\
\hline I will continue taking this brand & $.381^{*+}$ & $.341^{+\infty}$ & $293^{*+}$ & .068 & $.230^{-4}$ & $.194^{*+}$ & .081 \\
\hline Are you kidding me? & $.504^{+\infty}$ & $.432^{+*}$ & $.262^{*+}$ & $.173^{\text {th }}$ & $.224^{-{ }^{-}}$ & $.180^{\circ+}$ & $.249^{\circ-}$ \\
\hline No need to say more & $.595^{+\infty}$ & $.448^{+*}$ & $375^{+\infty}$ & $.38 g^{m+n}$ & $373^{*-4}$ & $.223^{++}$ & $.356^{+-}$ \\
\hline Confirmed / Total & $15 / 15$ & $15 / 15$ & $14 / 15$ & $9 / 15$ & $11 / 15$ & 9/15 & $11 / 15$ \\
\hline
\end{tabular}

**. Correlation is significant at the 0.01 level (2-tailed).

*. Correlation is significant at the 0.05 level (2-tailed).

Table 1 shows the relationship between positive comments written in different ways and the plain unformatted text. Plain unformatted text refers to the texts that are written without any capital little, repetition words, emoticons or any strengthening words.

The results from table 1 shown that $\mathrm{H} 1$ is fully supported. All of the comments written in capital letters (e.g. I LOVE IT) have significant relationship with its original text (e.g. I love it). This explains that when the whole comment is written in all capital letters, it has significantly influenced its comments expression level. H2 is also fully supported. Phrases that end with less than 4 exclamation marks (e.g. I love it!!), have significant p-values that show that its expressional level is different from the way it is written originally.

H3 expression intention of phrase with more than 4 exclamation marks is also significantly affected. However, the value of each comment on $\mathrm{H} 3$ to compare to $\mathrm{H} 2$, has yet to be identified in this paper. $\mathrm{H} 4$ is also supported. Nine out of its 15 comments show its significant value. Further analysis is to be performed with statistic identification comparing to its negative significant value. Comments with repeated letters (e.g. I loooooove you) also show significant effects on expression intention, tests show 11 out of 15 are positive. Comments ending with positive emoticon (e.g. :-)) has effected its expression intention compared to the original written text without emoticon. We have yet to investigate how sarcastic use of emoticons with positive and negative comments. However, positive comments with negative emoticons (e.g. I love it :-( ) have higher significant effects compared to the positive emoticons.

\section{Table 2 Correlation of negative comments}

\begin{tabular}{|l|c|c|c|c|c|c|c|}
\hline Comment & H1 & H2 & \multicolumn{1}{|c|}{ H3 } & H4 & H5 & H6 & H7 \\
\hline Some serious abuse & $.416^{* *}$ & $.439^{* *}$ & $.477^{* *}$ & $.331^{* *}$ & $.357^{* *}$ & -.073 & $.284^{* *}$ \\
\hline Very disappointed & $.404^{* *}$ & $.418^{* *}$ & $.342^{* *}$ & $.121^{* *}$ & -.010 & $-.150^{* *}$ & .087 \\
\hline I don't care & $.561^{* *}$ & $.437^{* *}$ & $.566^{* *}$ & $.403^{* *}$ & $.237^{* *}$ & .020 & $.432^{* *}$ \\
\hline I did hit it well & $.491^{* *}$ & $.455^{* *}$ & $.455^{* *}$ & $.458^{* *}$ & $.372^{* *}$ & $.093^{*}$ & $.192^{* *}$ \\
\hline I hate it & $.691^{* *}$ & $.644^{* *}$ & $.582^{* *}$ & $.393^{* *}$ & $.387^{* *}$ & -.065 & $.377^{* *}$ \\
\hline It is really annoying & $.621^{* *}$ & $.630^{* *}$ & $.511^{* *}$ & $.398^{* *}$ & $.148^{* *}$ & .075 & $.430^{* *}$ \\
\hline I boot it & $.574^{* *}$ & $.286^{* *}$ & $.319^{* *}$ & .041 & .067 & .054 & $.190^{* *}$ \\
\hline Too much trouble & $.511^{* *}$ & $.433^{* *}$ & $.384^{* *}$ & $.226^{* *}$ & $.120^{* *}$ & -.034 & $.203^{* *}$ \\
\hline Totally fierce & $.647^{* *}$ & $.548^{* *}$ & $.485^{* *}$ & $.276^{* *}$ & $.230^{* *}$ & $-.173^{* *}$ & .082 \\
\hline I have to worry & $.630^{* *}$ & $.534^{* *}$ & $.574^{* *}$ & $.345^{* *}$ & $.411^{* *}$ & $.111^{*}$ & $.409^{* *}$ \\
\hline I can afford it & $.358^{* *}$ & $.347^{* *}$ & $.255^{* *}$ & .076 & $.137^{* *}$ & $.204^{* *}$ & $.231^{* *}$ \\
\hline What a lie & $.596^{* *}$ & $.381^{* *}$ & $.417^{* *}$ & $.284^{* *}$ & $.310^{* *}$ & $-.103^{*}$ & $.256^{* *}$ \\
\hline Don't come here to shop & $.679^{* *}$ & $.517^{* *}$ & $.495^{* *}$ & $.416^{* *}$ & $.364^{* *}$ & $.114^{*}$ & $.280^{* *}$ \\
\hline Fine until it breaks & $.546^{* *}$ & $.537^{* *}$ & $.485^{* *}$ & $.247^{* *}$ & $.211^{* *}$ & $.164^{* *}$ & $.261^{* *}$ \\
\hline Never, ever, never & $.676^{* *}$ & $.600^{* *}$ & $.561^{* *}$ & $.298^{* *}$ & $.411^{* *}$ & .014 & $.297^{* *}$ \\
\hline Confirmed/ Total & $15 / 15$ & $15 / 15$ & $15 / 15$ & $13 / 15$ & $13 / 15$ & $8 / 15$ & $13 / 15$ \\
\hline
\end{tabular}

Table 2 presents the relationship between negative comments written in different ways compared to its plain unformatted text. H1 is fully supported. The words "IT IS REALLY ANNOYING" has effect of expression toward plain text "It is really Annoying". Again, phrases with less than four exclamation marks (e.g. It is really annoying!!) influences emotional intention compared to text without exclamation marks. H2 supported with 15 of 15 cases. Similarly, comments with more four exclamation marks (e.g. It is really annoying!!!!!) has a significant impact on the expression intention, with 15 of 15 cases. H4 is supported in 13 out of 15 cases. It is high and it shows that negative comment with certain word in capital letter (e.g. It is really ANNOYING) has it significant effects on its expression intention. $\mathrm{H} 5$ is confirmed with 13 out of 15 cases, it also explains that negative comment with repeated alphabet letter (e.g. It is really annoooooooying) effects emotional level of intention. Negative comment with positive emoticon (e.g. It is really annoying:-) ) however, has lower results 9 out of 15 only, which also meant that postive emoticons that come along with negative 
comments has lower significance comparing to using capital letters or exclamation marks. H6 is logical, it can be sarcastic expression but still in negative value. Further work would need to be conducted on the effect of sarcasm. Lastly, negative comments with negative emoticon has also shown its effects expression intention with the result of 13 out of 15 .

\section{CONCLUSION AND FUTURE WORK}

Overall, we have shown that there are significant interpretation effects of text formatting, punctuation and emoticons on both positive and negative comments. Significant features include 1) comments written in capital letters 2) comments that end with fewer exclamations marks, 3 ) comments that end with more than 4 exclamation marks, 4) comments containing certain words in capital letters even though it is less significant compared to H4, and 5) comments comes with repetition in letters, 6) comments that end with positive emoticon, and lastly 7) comments that end with negative emoticon. Although the comments with specific words in capital letters have less significant correlation than the others, it still has more than half of its comments supported with its significant level. Further work remains to be done to take into account sarcasm, although we expect this to be a very challenging problem. Compared to negative comments, positive comments have lesser number of significant cases. However, when it is ended with negative emoticon, a positive comment shows higher number of is significant. We will explore these features in our future studies.

\section{REFERENCES}

[1] M. Khoury and S. Shirmohammadi, "Accessibility and scalability in collaborative eCommerce environments," 2007 2nd Int. Conf. Digit. Inf. Manag. ICDIM, vol. 2, 2007.

[2] S. Lin and S. Yan, "Taobao data on women's apparel online sales of consumer behavior analysis," $2011 \mathrm{Int}$. Conf. Internet Technol. Appl. iTAP 2011 - Proc., no. July 2010, pp. 1-4, 2011.

[3] Z. Shen and N. Sundaresan, "eBay : An E-Commerce Marketplace as a Complex Network," Wsdm, pp. 655664, 2011.

[4] M. . Hossain, "“Performance Evaluation Web Testing For ECommerce Website,"” Informatics, Electron. Vis. (ICIEV), 2012 Int. Conf., pp. 842-846, 2012.

[5] G. He, Y. Deng, and X. Wu, "Analysis of web-surfing behavior of enterprise network users," Proc. 2nd Int. Conf. Comput. Sci. Netw. Technol. ICCSNT 2012, pp. 916-920, 2012.

[6] T. T. Zin, P. Tin, T. Toriu, and H. Hama, "Visual behavior analysis tool for consumer video surveillance," 1st IEEE Glob. Conf. Consum. Electron. 2012, GCCE 2012, vol. 1, no. 2, pp. 718$719,2012$.

[7] W. Fan and S. Sun, "Sentiment classification for online comments on Chinese news," ICCASM 2010 2010 Int. Conf. Comput. Appl. Syst. Model. Proc., vol. 4, no. Iccasm, pp. 740-745, 2010.

[8] D. Kim, M. G. Frank, and S. T. Kim, "Emotional display behavior in different forms of Computer Mediated Communication," Comput. Human Behav., vol. 30, pp. 222-229, 2014.

[9] I. Vandergriff, "Emotive communication online: A contextual analysis of computer-mediated communication (CMC) cues," J. Pragmat., vol. 51, pp. 1-12, 2013.

[10] A. Neviarouskaya, H. Prendinger, and M. Ishizuka, "Affect Analysis Model: novel rule-based approach to affect sensing from text," Nat. Lang. Eng., vol. 17, no. 01, pp. 95-135, 2011.

[11] B. Pang, L. Lee, and S. Vaithyanathan, "Thumbs up? Sentiment classification using machine learning techniques," Proc. Conf. Empir. Methods Nat. Lang. Process. July 6-7, 2002, Philadephia, Pennsylvania, USA, pp. 79-86, 2002.

[12] M. Thelwall and D. Wilkinson, "Public Dialogs in Social Network Sites: What Is Their Purpose?," J. Am. Soc. Inf. Sci. Technol., vol. 61, no. 2, pp. 392-404, 2010 .

[13] R. Prabowo and M. Thelwall, "Sentiment analysis: A combined approach," J. Informetr., vol. 3, pp. 143$157,2009$.

[14] M. Thelwall, K. Buckley, and G. Paltoglou, "Sentiment strength detection for the social web," $J$. Am. Soc. Inf. Sci. Technol., vol. 63, no. 1, pp. 163173, 2012.

[15] N. K. Cobb, D. Mays, and A. L. Graham, "Sentiment analysis to determine the impact of online messages on smokers' choices to use varenicline," J. Natl. Cancer Inst. - Monogr., no. 47, pp. 224-230, 2013.

[16] B. Xue, C. Fu, and Z. Shaobin, "A Study on Sentiment Computing and Classification of Sina Weibo with Word2vec," 2014 IEEE Int. Congr. Big Data, no. 2013, pp. 358-363, 2014.

[17] P.-W. Liang and B.-R. Dai, "Opinion Mining on Social Media Data," 2013 IEEE 14th Int. Conf. Mob. Data Manag., pp. 91-96, 2013. 
[18] Y. M. Kalman and D. Gergle, "052 Cmc Cues Enrich Lean Online Communication: the Case of Letter and Punctuation Mark Repetitions," MCIS 2010 Proc., p. 45, 2010.

[19] Y. Urabe, R. Rzepka, and K. Araki, "Emoticon Recommendation System for Effective Communication," Proceedings of the 2013 IEEE/ACM International Conference on Advances in Social Networks Analysis and Mining. 2013, pp. 1460-1461.
[20] Y. M. Kalman and D. Gergle, "Letter repetitions in computer-mediated communication: A unique link between spoken and online language," Comput. Human Behav., vol. 34, pp. 187-193, 2014.

[21] P. Rayson, "From key words to key semantic domains," J. of Corpus linguistic. vol. 4, no. 2008, pp. 519-549, 2008. 